\title{
BMJ Open Evaluation of the accuracy in detecting cervical lesions by nurses versus doctors using a stationary colposcope and Gynocular in a low-resource setting
}

Ashrafun Nessa, ${ }^{1}$ Joya Shree Roy, ${ }^{2}$ Most Afroza Chowdhury, ${ }^{1}$ Quayuma Khanam, ${ }^{1}$ Romena Afroz, ${ }^{1}$ Charlotte Wistrand, ${ }^{3}$ Marcus Thuresson, ${ }^{4}$ Malin Thorsell, ${ }^{3}$ Isaac Shemer, ${ }^{5}$ Elisabeth Andrea Wikström Shemer ${ }^{3}$

To cite: Nessa A, Roy JS, Chowdhury MA, et al. Evaluation of the accuracy in detecting cervical lesions by nurses versus doctors using a stationary colposcope and Gynocular in a low-resource setting. BMJ Open 2014;4: e005313. doi:10.1136/ bmjopen-2014-005313

- Prepublication history for this paper is available online. To view these files please visit the journal online (http://dx.doi.org/10.1136/ bmjopen-2014-005313).

Received 21 March 2014 Revised 9 October 2014 Accepted 16 October 2014

CrossMark

For numbered affiliations see end of article.

Correspondence to Dr Elisabeth Wikström Shemer;

elisabeth@gynocular.com

\section{ABSTRACT}

Objectives: Evaluation of the performance of VIA (visual inspection with acetic acid) trained nurses to learn colposcopy and the Swede score method to detect cervical lesions by using stationary colposcope or a portable, hand-held colposcope; the Gynocular, as compared to doctors.

Design: A crossover randomised clinical trial. Setting: The Colposcopy Clinic of Bangabandhu Sheikh Mujib Medical University (BSMMU), Dhaka, Bangladesh.

Participants: 932 women attending the clinic as either screening naïve for VIA screening (404) or women referred as VIA positive (528) from other VIA screening centres in the Dhaka region.

Intervention: VIA trained nurses were trained on-site in colposcopy and in the Swede score systematic colposcopy method. The Swede score grade cervical acetowhiteness, margins plus surface. vessel pattern, lesion size and iodine staining. The women were randomised to start the examination by either a stationary colposcope or the Gynocular. Swede scores were first obtained by a nurse and the same patient was equally evaluated by a doctor.

Primary and secondary outcome measures: Agreement between nurses and doctors in Swede scores was evaluated using the weighted $\kappa$ statistic for the Gynocular and standard colposcope. The ability to predict CIN 2+ (CIN 2, CIN 3 and invasive cervical cancer) using Swede scores was evaluated using receiver-operating characteristic curves.

Results: The Swede scores obtained by nurses and doctors using the Gynocular and stationary colposcope showed high agreement with a $\kappa$ statistic of 0.858 and 0.859 , respectively, and no difference in detecting cervical lesions in biopsy. Biopsy detected CIN $2+$ in $39(4.2 \%)$ women.

Conclusions: Our study showed that VIA nurses can perform colposcopy. There was no significant differences compared to doctors in detecting cervical lesions by stationary colposcope or the Gynocular using the Swede score system. Swede scores obtained

\section{Strengths and limitations of this study}

- The main strength of our study is its randomised crossover design including both screening naïve women and women referred as visual inspection with acetic acid (VIA positive), thus giving the examiners a wide range of normal to pathological colposcopic impressions, and a reduction of the risk of selection bias. The crossover randomised design was used to reduce the risk of intraobserver variability. Another strength is that all the biopsies were analysed in a single-site laboratory.

- The main limitation of our study is that not all the women examined had a biopsy, which may have biased our results. The crossover design may also have influenced the scoring of the second instrument.

- Other study designs were considered but would have been difficult to implement in a lowresource setting where many poor women might never return to the colposcopy clinic. Also, blinding of the instrument that was used was not possible due to the nature of the instruments. However, by using a crossover study design, block randomisation and the large sample size, we consider the risk of bias reduced.

by nurses using the Gynocular could offer an accurate cervical diagnostic approach in low resource settings.

Trial registration number: ISRCTN53264564.

\section{INTRODUCTION}

Cervical cancer is a common cause of death in women from low-resource settings. Recent data indicate that each year 528000 women are diagnosed with cervical cancer worldwide and 266000 die from the disease. ${ }^{1} \mathrm{~A}$ 
majority $(87 \%)$ of women diagnosed with cervical cancer live in less developed regions of the world. ${ }^{1}$ Mortality varies highly, ranging from less than 2/100 000 in developed regions to more than 20/100 000 in areas such as Melanesia and Middle and Eastern Africa. ${ }^{1}$

In Bangladesh, 11956 new cases of cervical cancer are discovered yearly and each year 6582 women die from the disease. ${ }^{1}$ Moreover, as the incidence rises sharply among women over 35 years of age, cervical cancer has a vast impact on communities at a time when a woman's presence is vital to social and economical stability. ${ }^{2}{ }^{3}$ According to the International Agency for Research on Cancer (IARC's) projections for the coming decades, deaths from cervical cancer will continue to rise. ${ }^{1}$

In high-resource settings, cervical cytology screening programmes have successfully reduced the rates of cervical cancer. ${ }^{4}$ In many low-resource settings, few women have access to cytology screening programmes due to the absence of national screening programmes, lack of equipment and skilled technicians. ${ }^{5}$ As a result, other low-cost methods have been implemented for screening, where opportunistic visual inspection with acetic acid (VIA) by trained nurses and doctors is the most common approach. ${ }^{6-8}$ Often, VIA positive women are referred for colposcopy in order to reduce the risk of overtreatment. $^{6-9}$

The role of colposcopy is to visually assess the size and extent of cervical lesions with strong illumination and magnification, and to select the most abnormal lesions for biopsy. ${ }^{10}$ In low-resource settings, colposcopy is often used to assess the severity of the lesion in VIA positive women as part of a 'see and treat' policy, as it may be more practical to treat the women immediately if a severe lesion is diagnosed, as she might not be able to return to the clinic. ${ }^{11}$

Colpscopy may also be used for primary screening in low-resource settings, especially when using the Swede score systematic colposcopy system, where the total score gives an indication of the severity of the visual impression of the cervix. ${ }^{11-15}$ However, the limited access of doctors and the limitations of stationary colposcopes (heavy, requires an electrical grid and technical support) are reasons why screening colposcopy has not reached widespread use in rural areas with poor infrastructure. ${ }^{11} 121415$

In high-resource settings, nurse colposcopists have been trained to increase accessibility to colposcopy and achieve similar diagnostic accuracy as doctors, ${ }^{16-18}$ and a similar approach in low-resource settings, with diagnosis and screening by the portable battery-driven colposcope, the Gynocular, could offer an accurate, cost-effective and pragmatic approach to combat cervical cancer. ${ }^{13-15}$ By using the Swede score systematic colposcopy method, the findings of the colposcopic findings of the cervix can be organised and scored as a structured report of the colposcopic examination. ${ }^{12}{ }^{13}$ The Swede score differs from other cervical scoring systems by adding the lesion size as a variable. ${ }^{12} 13$

The main aim of this study was to evaluate if nurse-led Swede score colposcopy in a low-resource setting had a similar performance to that of a doctor Swede score colposcopy in detecting cervical lesions. The second aim of the study was to evaluate if a nurse or doctor Gynocular colposcopy had similar performances as compared to a stationary colposcopy in detecting cervical lesions. The third aim of the study was to evaluate if nurse-led Swede score colposcopy could be a future option for detection of cervical lesions in low-resource settings.

\section{METHODS}

\section{Design, participants and procedures}

This study was a randomised crossover clinical trial for evaluating the accuracy of Swede scores of VIA nurses trained in the Swede score colposcopy method in detecting cervical lesions using biopsy as a criterion standard. All the participating women were randomised to be examined using both the stationary colposcope and the Gynocular in a crossover design. The nurses and the doctors examined the same women, but were blinded from each other's Swede scores.

In Bangladesh, $2.3 \%$ of the women have been screened with VIA so far, and among those who have been screened, approximately $4.8 \%$ were VIA positive. ${ }^{6}$ The opportunistic VIA screening programme in Bangladesh was initiated in the year 2004 by the government of Bangladesh. Trained paramedics, senior staff nurses and doctors offer VIA to married women 30 years and above to detect the precancer and early cervical cancer among women visiting VIA centres of different districts in Bangladesh. ${ }^{18-20}$ VIA-positive women are referred to BSMMU and government medical college hospitals for colposcopic evaluation and management. ${ }^{18-20}$ The cervical cancer screening programme is an ongoing programme and colposcopy becomes an important part of this prevention programme. A woman is considered to be VIA positive when sharp, distinct, well-defined, dense acetowhite areas on the cervix are noticed during examination with or without raised margins, close to the squamocolumnar junction in the transformation zone. ${ }^{6} 8$

The inclusion criteria were: (1) women positive for VIA at opportunistic screening by trained family welfare visitors, senior staff nurses and doctors in the Dhaka region, Bangladesh referred for colposcopy, or women coming for VIA cervical screening at the colposcopy clinic of Bangabandhu Sheikh Mujib Medical University (BSMMU) during 1 June to 31 September 2012. Other inclusion criteria were: (2) ability to understand written and oral information, (3) women signing an informed consent form to participate in the study after receiving oral and written information from a social worker. Exclusion criteria were: (1) ongoing vaginal bleeding, (2) any previous gynaecological examinations less than 1 week (3) pregnancy. Women who chose not to take part in the study had a standard colposcopy examination.

In total, 932 women were included in the study, of them 404 attending the clinic as screening naive and 528 women referred as VIA positive. Two VIA nurses 
were trained on-site in colposcopy and the Swede score systematic colposcopy method for 2 weeks before starting the study. The colposcopy specialists were accredited physicians or gynaecologists who were trained in colposcopy, cold coagulation and the loop electrical excision procedure at the colposcopy clinic of BSMMU. ${ }^{7}$ All women in the study were examined by one of the two nurses and one of the six colposcopy specialists and both by the stationary colposcope and the Gynocular.

During the colposcopy examination, one of the two standard colposcopes (Leisegang 1DF, Leisegang, Feinmechanik-Optik GmbH, Berling, Germany or Karl Kaps Som 52, Karl Kaps GmbH \& Co.KG, Asslar/Wetzlar, Germany) and the Gynocular (Gynius AB, Stockholm, Sweden) were used. Women were randomly allocated in blocks of 50 to start the examination by one of the nurses, followed by an examination by one of the doctors with either the stationary colposcope or the Gynocular. Then the same examiners examined the woman with the second instrument in order to assess the performance of agreement between the Swede scores of the nurse and of the doctor and of the two instruments. The crossover design was chosen in order to lessen possible observer variability. ${ }^{21} \mathrm{~A}$ total of 524 women started the examination with the stationary colposcope and 408 women with the Gynocular. During the Swede score examination, each of the five colposcopic variables (acetowhiteness, margins plus surface, vessel pattern, lesion size and iodine staining) was given a score of 0,1 or 2 points. ${ }^{12-15}$

A self-holding non-lubricated speculum was placed in the vagina and the cervix was visualised. The nurse started the examination with an inspection of the cervical vessels of mosaic pattern, punctuation, atypical vessels or absence of vessels as randomised with the colposcope or the Gynocular using the red-free (green filter) mode and switched instruments. Then the doctor evaluated the vessel patterns with both instruments as randomised. This procedure differs slightly from the original Swede score, ${ }^{12}$ where the vessels are inspected after application of acetic acid to the cervix. However, after application of acetic acid, the whitening effect on the cervix slowly fades. Therefore, we chose to first inspect the vessel pattern, and then apply the acetic acid to ensure that both nurses and doctors would have enough time to accurately estimate the acetowhitening effect before fading. Thus, the cervix was wiped with $5 \%$ acetic acid for $1 \mathrm{~min}$, followed by evaluation by the nurse of three Swede score variables (acetowhiteness, margins plus surface and lesion size) and scored by the nurse by both instruments and then again by the doctor. Next, the cervix was swabbed with 5\% Lugol iodine solution, and the nurse scored the Swede score's fifth variable (iodine staining) with both instruments as randomised and then again by the doctor. The results from the examinations were reported by the nurses and the doctors to the study nurse in a separate location. The other examiners could not overhear the reports and they could not see the results of the previous examiner. The nurses and the doctors were also not allowed to communicate the patient's examination results. The examination was completed with one or more biopsies taken from areas of suspected cervical lesions. Punch biopsies of the cervix were performed in all women in whom the doctor had scored a Swede score $\geq 4{ }^{12} 1315$ as doctors using the Swede score have been validated and biopsy recommended when Swede scores are 6 and above, ${ }^{12} 13$ and this study aimed to validate the nurse colposcopist's Swede scoring in a lowresource setting (which has not been validated before). The cervical biopsies were analysed at the histopathology laboratory of BSMMU. The histopathology diagnoses were graded according to the CIN classification system and considered as the gold standard. ${ }^{22}$

Women with CIN1 lesions were given the choice of direct treatment or a follow-up examination after 6 months. Women with CIN2+ were offered a loop electrical excision procedure. Women with invasive cervical cancers were referred to the Gynecological Oncology Unit of the Department of Obstetrics and Gynaecology at BSMMU for management. This study was approved by the local ethics committees in Bangladesh and in Sweden: the Institutional Review Board of BSMMU; Dnr BSMMU/2012/3176 and the Stockholm Regional Ethical Review Board; Dnr 2012/545-31/1. The study was registered as ISRCTN53264564 at http://www. controlled-trials.com after enrolment of the participants started, due to the publishing restraints of the parallel patenting process of the Gynocular.

The study protocol can be accessed at the Department of Obstetrics and Gynecology, Danderyd Hospital, Stockholm, Sweden.

The Gynocular (Gynius AB, Stockholm, Sweden) is a high resolution monocular colposcope with similar specifications to stationary colposcopes. ${ }^{14}{ }^{15}$ The Gynocular is a small, hand-held, battery-driven, measuring 50×33×166 $\mathrm{mm}$ with $300 \mathrm{~mm}$ focal distance, and three magnifications: $5 \times, 8 \times$ and $12 \times{ }^{14}{ }^{15}$ It has a tripod mounting clip that screws into any standard tripod, allowing the medical professional to perform colposcopy in a hands-free mode for ease of biopsy (figure 1). The Gynocular has high-intensity light-emitting diodes for warm white illumination, a green filter light, and is powered by a rechargeable lithium-ion battery. It is a product approved by the Swedish National Drug Authority as a non-invasive medical diagnostic class I tool, CE marked and Food and Drug Administration approved.

\section{Statistical analysis}

All statistical analyses have been performed using $\mathrm{R}$ V.2.14. ${ }^{23}$ The baseline patient characteristics of the women were summarised using means and SD for continuous variables and absolute and relative frequencies for categorical variables. To test the level of agreement between the colposcope and the Gynocular, the percentage agreement and the weighted $\kappa$ statistic was calculated. ${ }^{24}$ Cervical lesions were classified by the Swede scores system using the Gynocular and the stationary colposcope. ${ }^{12-15}$ Detection rates of CIN 1, CIN 2, CIN 3, 


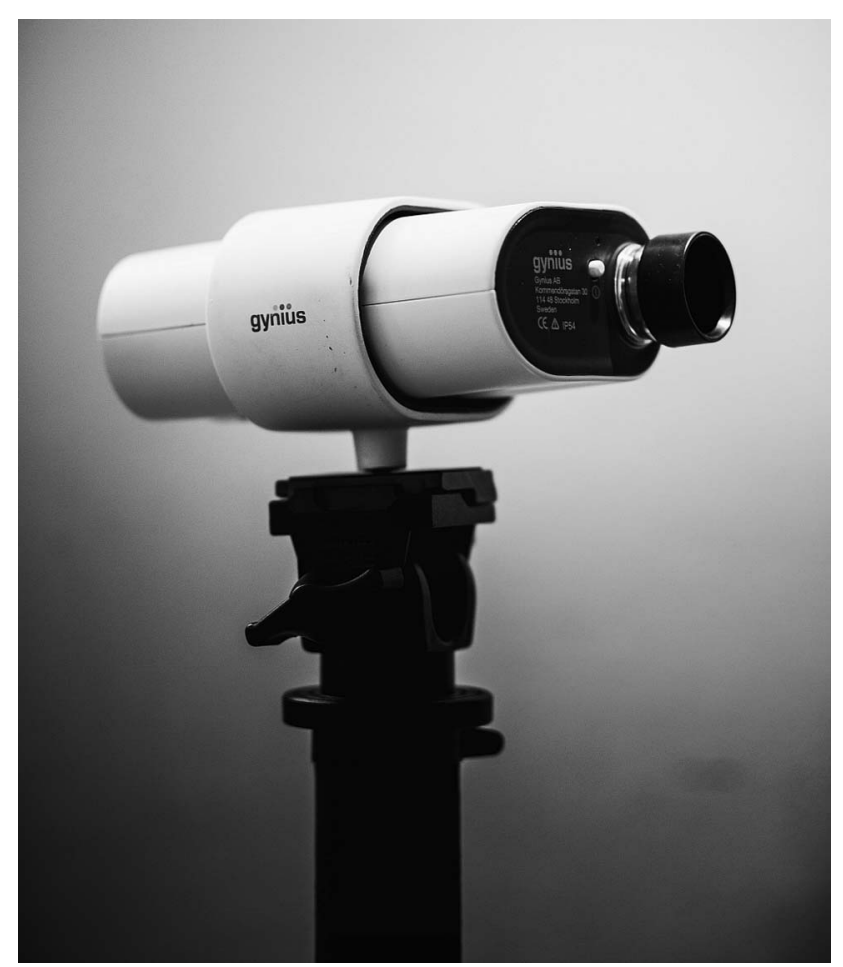

Figure 1 The Gynocular with a tripod mounting clip that screws into any standard tripod.

ICC (invasive cervical cancer), AIS (adenocarcinoma in situ), benign cervicitis and cervical tuberculosis in cervical punch biopsies were calculated. A positive biopsy result was defined as CIN2+ (CIN 2, CIN 3, CIN 3+) and we calculated the Swede score's sensitivity, specificity, positive predictive value (PPV) and negative predictive value (NPV) using biopsy as a gold standard for all cut-off levels of Swede scores between 4 and 10 for doctors and 0 and 10 for nurses. The results are presented in tables and as receiver operating characteristic (ROC) curves as well as the area under the curve (AUG). The comparison of AUC of the ROC curves was performed using the roc.test function in the pROC package.

\section{Sample size}

The sample size was estimated based on the expected number of positive biopsy results (defined as CIN2+) and not in terms of statistical power. In Bangladesh, there are no published data on CIN2+ in a previously unscreened population in Bangladesh verified by cytology, colposcopy and biopsy, only of CIN2+ in VIA positive women. However, data from India show a rate of $2.7 \%$ CIN2+ in unscreened women. ${ }^{25}$ Thus, we assumed a similar rate in Bangladesh with an expected rate of CIN2+ of $2.5 \%$ in naïe women and $7.5 \%$ in VIA-positive women as a sample size of 500 naïve and 500 VIA-positive women would generate 50 positive biopsy results, which were considered to give sufficient precision to the nurses colposcopists' accuracy and Swede scoring compared to doctors. ${ }^{12} 13$ Thus, the aim was to include a total of approximately 1000 women.
In a retrospective power analysis based on the results from the present study, we estimated that approximately 1500 biopsies would have been needed (as compared to the 228 biopsies in women with a Swede score above 4 in this study) for $80 \%$ power to detect a difference of 0.05 in the AUC of the ROC curves at a $5 \%$ significance level.

\section{RESULTS}

A total of 932 women were included in the study, of which $404(43 \%)$ were screening naïve. The women's baseline characteristics are presented in table 1. A total of 256 women had a Swede score of at least 4 by a doctor, and of them 228 had a biopsy and 28 refused biopsy (excluded from the ROC analyses). Fifty-nine biopsies were taken outside the research protocol (excluded from ROC analyses). Twenty-seven VIA positive women had CIN2+ and 5 screening naïve women had CIN2+. Punch biopsy was benign in $7(1.8 \%)$, chronic cervicitis in $23(5.8 \%)$, CIN1 in $19(4.8 \%)$ and CIN2 in $4(1.0 \%)$. No women had CIN3 and $4(1.0 \%)$ had ICC (CIN3+). In $1(0.2 \%)$, the woman's biopsy showed tuberculosis. The Swede score was $<4$ in 342 $(85.5 \%)$ women, and in those women no biopsy was taken.

Among the referred VIA-positive women, punch biopsy was benign in $13(2.5 \%)$, chronic cervicitis in 82 $(15.7 \%)$, CIN1 in $90(17.3 \%)$ and CIN2 in $21(4 \%)$. Four women $(0.8 \%)$ had CIN3 and $6(1.2 \%)$ had ICC (CIN3+). Two $(0.4 \%)$ women had tuberculosis in the biopsy. In $303(57.4 \%)$ women, the Swede score was $<4$ and no biopsy was taken.

When cross tabulating Swede scores by the Gynocular of nurses and doctors, the $\kappa$ coefficient was $0.859, \mathrm{p}$ value $<0.001$ (figure 2) and Swede scores by the colposcope by nurses and doctors had a $\kappa$ coefficient of 0.858 , p value $<0.001$ (figure 3 ) .

Cross tabulation of Swede scores by the colposcope versus the Gynocular by nurses showed a $\kappa$ coefficient of 0.997 ( $\mathrm{p}$ value $<0.001$ ), and a cross tabulation of Swede scores by the colposcope and the Gynocular for the doctors showed a $\kappa$ coefficient of 0.998 ( $p$ value $<0.001$ ).

There were no significant differences between the Swede scores of the nurses and the doctors in predicting a positive biopsy result (CIN2+) for both the Gynocular (figure 4) and the colposcope (figure 5).

With a cut-off value of 6 and above for Swede score and biopsy, Gynocular by nurses had a sensitivity of $52.8 \%$ $(95 \%$ CI $35.5 \%$ to $69.6 \%)$ and a specificity of $65.6 \%$ (95\% CI $58.4 \%$ to $72.4 \%$ ) for CIN2+ and stationary colposcope by nurses had a sensitivity of $52.8 \%(95 \%$ CI $35.5 \%$ to $69.6 \%)$ and a specificity of $66.1 .6 \%(95 \%$ CI Q14 $58.9 \%$ to $72.8 \%$ for CIN2+ (table 2). For doctors using the Gynocular and having a cut-off value of 6 , the sensitivity was $61.1 \%$ (95\% CI $43.5 \%$ to $76.9 \%)$ and specificity $52.9 \%$ (95\% CI $45.5 \%$ to $60.1 \%)$, and for the stationary colposcope the sensitivity was $61.1 \%$ (95\% CI $43.5 \%$ to $76.9 \%$ ) and specificity $53.4 \%$ (95\% CI $46.1 \%$ to $60.6 \%$ ) for detecting CIN2+ (table 3). The sensitivity decreased while specificity increased with the increased 
Table 1 Baseline characteristics

\begin{tabular}{|c|c|c|c|}
\hline & & $\begin{array}{l}\text { Screening naive } \\
(\mathrm{N}=404)\end{array}$ & $\begin{array}{l}\text { VIA positive } \\
(\mathrm{N}=528)\end{array}$ \\
\hline Age & Mean (SD) & $35.1(8.1)$ & $34.1(7.8)$ \\
\hline Age when married & Mean (SD) & $17.9(4.5)$ & $17.1(3.6)$ \\
\hline Age at first delivery & Mean (SD) & $20.0(4.4)$ & $19.2(3.5)$ \\
\hline Parity & n (\%) & & \\
\hline 0 & & $14(3.5)$ & $12(2.3)$ \\
\hline 1 & & $82(20.3)$ & $96(18.5)$ \\
\hline 2 & & $163(40.4)$ & $216(41.5)$ \\
\hline 3 & & $83(20.6)$ & 102 (19.6) \\
\hline 4 & & $40(9.9)$ & $57(11.0)$ \\
\hline 5 & & $17(4.2)$ & $22(4.2)$ \\
\hline 6 & & $1(0.2)$ & $9(1.7)$ \\
\hline 7 & & $1(0.2)$ & $5(1.0)$ \\
\hline Over 8 & & $2(0.5)$ & $1(0.2)$ \\
\hline Biopsy results & n (\%) & & \\
\hline Benign & & $7(1.7)$ & $13(2.5)$ \\
\hline Chronic cervicitis & & $23(5.7)$ & $82(15.7)$ \\
\hline CIN1 & & $19(4.7)$ & 90 (17.3) \\
\hline CIN2 & & $4(1.0)$ & $21(4.0)$ \\
\hline CIN3 & & $0(0.0)$ & $4(0.8)$ \\
\hline ICC & & $4(1.0)$ & $6(1.2)$ \\
\hline Tuberculosis & & $1(0.2)$ & $2(0.4)$ \\
\hline No biopsy & & $343(85.5)$ & 303 (58.2) \\
\hline
\end{tabular}

Swede score for CIN2+, both for nurses and doctors, and with the increasing Swede scores, nurses had a higher sensitivity in the upper Swedes scores in detecting CIN2+ (tables 2 and 3). A Swede score of 8 and above had high specificity for CIN2+ lesions (tables 2 and 3).

We further subanalysed the nurses 50 first Swede scores for predicting CIN2+ (figure 6), where the specificity for high Swede scores was high, but sensitivity was lower than when the nurses had had further practice.

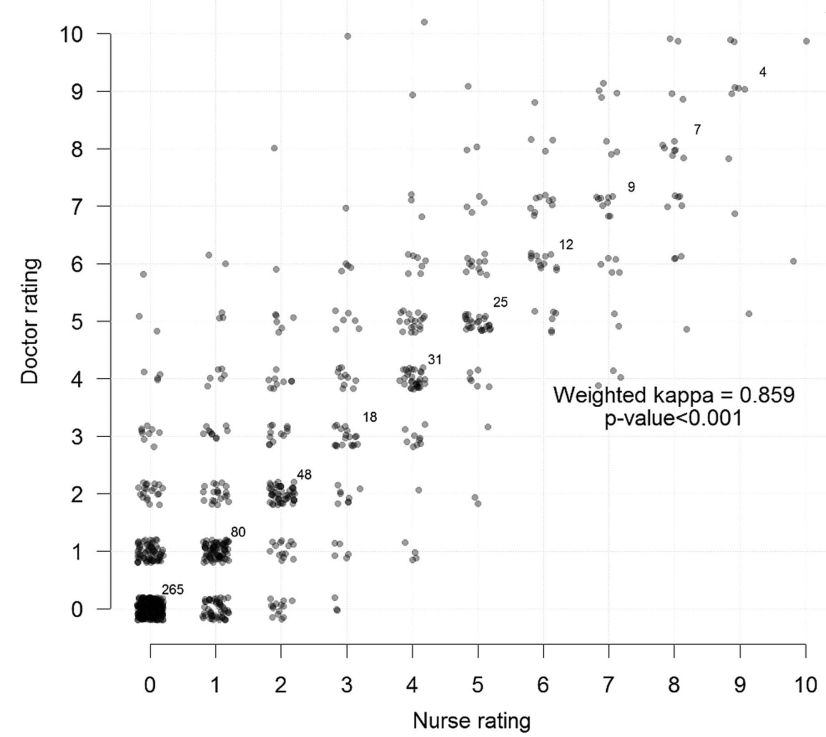

Figure 2 Cross tabulation of Swede scores by the Gynocular of nurses and doctors with $\kappa$.

\section{DISCUSSION}

This study found that VIA nurses trained in colposcopy in a low-resource setting detect high-grade cervical lesions as well as specialised doctors do using the Swede score systematic colposcopy system. Also, there were no significant differences for the nurses or the doctors using the Gynocular and the standard colposcope in detecting cervical lesions, confirmed by a high agreement of Swede scores and the histopathological diagnosis from punch

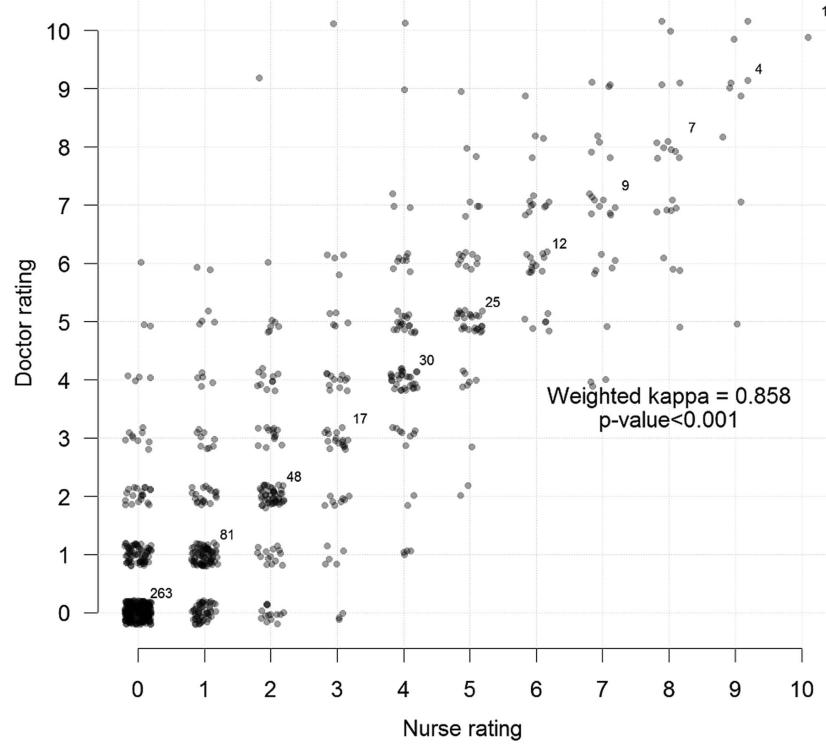

Figure 3 Cross tabulation of Swede scores by the stationary colposcope of nurses and doctors. 


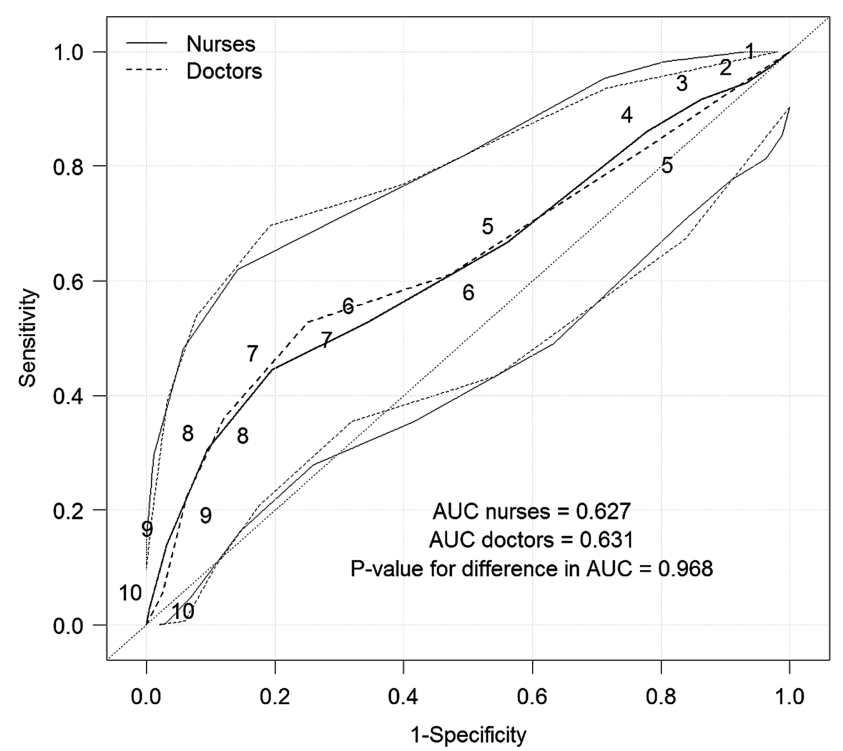

Figure 4 Receiver operating characteristic curves for predicting a positive biopsy result for $\mathrm{CIN} 2+(\mathrm{CIN} 2, \mathrm{CIN} 3$ and invasive cervical cancer) by the Gynocular and Swede scores of 1-10 for nurses and 4-10 for doctors (as the doctor's Swede score decided whether a biopsy was necessary).

biopsy. Moreover, Swede scores of 8 and above had high specificity for CIN2+ lesions.

\section{Strength and limitations}

The main strength of our study is its randomised crossover design including both screening naive women and women referred as VIA positive, thus giving the

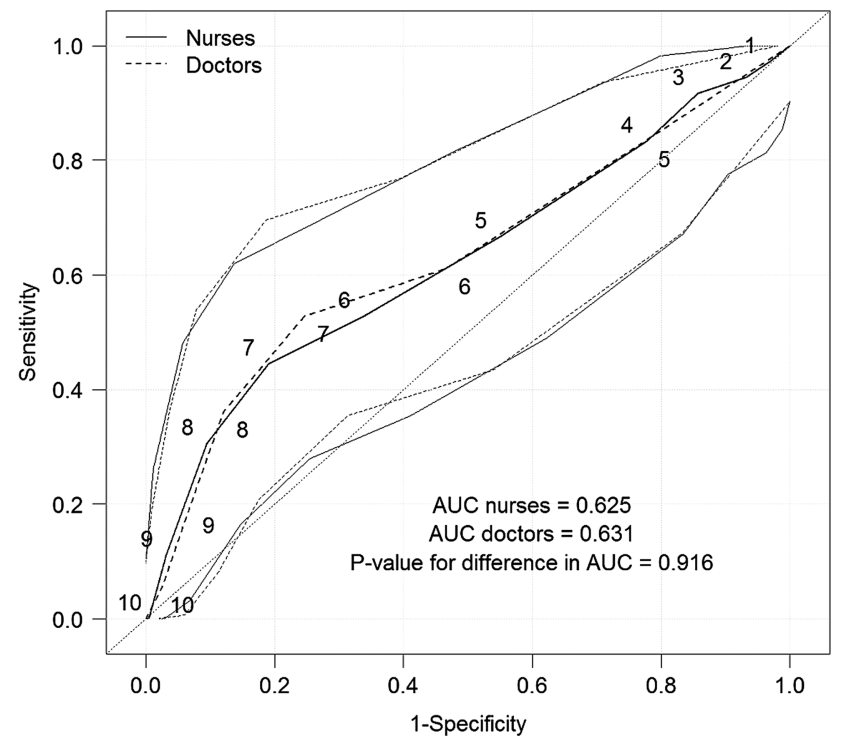

Figure 5 Receiver operating characteristic curves for predicting a positive biopsy result for $\mathrm{CIN} 2+(\mathrm{CIN} 2, \mathrm{CIN} 3$ and invasive cervical cancer) by a stationary colposcope and Swede scores of 1-10 for nurses and 4-10 for doctors (as the doctor's Swede score decided whether a biopsy was necessary). examiners a wide range of normal to pathological colposcopic impressions, and a reduction of the risk of selection bias. The crossover randomised design was used to reduce the risk of intraobserver variability. ${ }^{21}$ Other strengths are that all the biopsies were analysed in a single-site laboratory, and the large number of included women were all examined in a single centre.

The main weakness of our study is that not all the women examined had a biopsy, which may have biased our results. However, the Swede score has already been validated in Sweden and in the $\mathrm{UK}^{12}{ }^{13}$ and a cervical biopsy was recommended for a Swede score of 6 and above. The Swede score has also been used in previous Gynocular studies in other low-resource settings. ${ }^{14} 15$ In our study, we lowered the Swede score biopsy threshold to 4 , as we worked in a low-resource setting with limited resources for follow-up and call back service of the included women. Interestingly, even when lowering the threshold for biopsy, we found few VIA positive women with a CIN2+ lesion, results that are similar to the results of our previous studies. ${ }^{14} 15$ In screening naïve women, it was even more uncommon with CIN2+. It was reassuring to note that these women were detected by both nurses and doctors.

The crossover design was chosen to lessen possible observer variability, but may also have influenced the scoring of the second instrument. Other study designs were evaluated but would have been difficult to implement in a low-resource setting, where many poor women may never return to the colposcopy clinic. Also, blinding of the instrument that was used was not possible due to the nature of the instrument. However, by using a crossover study design, block randomisation and the large sample size, we reduced the risk of the second examination's possible influence of cervical impression to affect the statistical calculations. Further, the inclusion of postmenopausal women and possible breastfeeding women could have affected the results, as the Swede score has not been validated on postmenopausal women and breastfeeding women.

In low-resource settings, 535900 women die from obstetric and pregnancy-related conditions each year. ${ }^{26-28}$ This has led to that task-shifting within obstetrics has been widely embraced in Sub-Saharan Africa, to make best use of available human resources by redistributing specific tasks from highly qualified specialists to the least specialised professional skilled of performing the assignment safe and reliably, including general practitioners or nonphysician clinicians (NPC) such as nurses, midwives and surgical technicians. ${ }^{25}{ }^{26}$ NPCs perform caesarean section and instrumental delivery in several African countries, thus bridging the human resource gap. ${ }^{25} 26$

The task-shifting could also be applied to colposcopy since 266000 women die from cervical cancer each year, and a majority of them in low-resource settings. ${ }^{1}$ In many high-resource settings, nurse colposcopists are a well-established resource within colposcopy, ${ }^{16-18}$ and with the results from our study we show that task-shifting 
Table 2 Sensitivity and specificity for different cut-off levels for CIN 2+ (CIN 2, CIN 3 and invasive cervical cancer; nurses, $n=228)$

\begin{tabular}{|c|c|c|c|c|}
\hline & Sensitivity $(95 \% \mathrm{Cl})$ & Specificity (95\% Cl) & PPV (95\% Cl) & NPV (95\% Cl) \\
\hline \multicolumn{5}{|l|}{ Gynocular } \\
\hline 10 vs $<10$ & $2.8 \%$ (0.1\% to $14.5 \%)$ & $99.5 \%(97.1 \%$ to $100.0 \%)$ & $84.3 \%(78.9 \%$ to $88.8 \%)$ & $50.0 \%(1.3 \%$ to $98.7 \%)$ \\
\hline$\geq 9$ vs $<9$ & $13.9 \%(4.7 \%$ to $29.5 \%)$ & $96.8 \%(93.2 \%$ to $98.8 \%)$ & $85.5 \%(80.1 \%$ to $89.9 \%)$ & $45.5 \%(16.7 \%$ to $76.6 \%)$ \\
\hline$\geq 8$ vs $<8$ & $30.6 \%(16.3 \%$ to $48.1 \%)$ & $90.5 \%(85.4 \%$ to $94.3 \%)$ & $87.2 \%(81.7 \%$ to $91.6 \%)$ & $37.9 \%(20.7 \%$ to $57.7 \%)$ \\
\hline$\geq 7$ vs $<7$ & $44.4 \%(27.9 \%$ to $61.9 \%)$ & $80.4 \%(74.0 \%$ to $85.8 \%)$ & $88.4 \%(82.6 \%$ to $92.8 \%)$ & $30.2 \%(18.3 \%$ to $44.3 \%)$ \\
\hline$\geq 6$ vs $<6$ & $52.8 \%(35.5 \%$ to $69.6 \%)$ & $65.6 \%(58.4 \%$ to $72.4 \%)$ & $87.9 \%(81.4 \%$ to $92.8 \%)$ & $22.6 \%(14.2 \%$ to $33.0 \%)$ \\
\hline$\geq 5$ vs $<5$ & $66.7 \%(49.0 \%$ to $81.4 \%)$ & $43.9 \%(36.7 \%$ to $51.3 \%)$ & $87.4 \%(79.0 \%$ to $93.3 \%)$ & $18.5 \%(12.2 \%$ to $26.2 \%)$ \\
\hline$\geq 4$ vs $<4$ & $86.1 \%(70.5 \%$ to $95.3 \%)$ & $22.2 \%(16.5 \%$ to $28.8 \%)$ & $89.4 \%(76.9 \%$ to $96.5 \%)$ & $17.4 \%(12.2 \%$ to $23.8 \%)$ \\
\hline$\geq 3$ vs $<3$ & $91.7 \%(77.5 \%$ to $98.2 \%)$ & $13.8 \%(9.2 \%$ to $19.5 \%)$ & $89.7 \%(72.6 \%$ to $97.8 \%)$ & $16.8 \%(11.9 \%$ to $22.8 \%)$ \\
\hline$\geq 2$ vs $<2$ & $94.4 \%(81.3 \%$ to $99.3 \%)$ & $6.9 \%(3.7 \%$ to $11.5 \%)$ & $86.7 \%(59.5 \%$ to $98.3 \%)$ & $16.2 \%(11.5 \%$ to $21.9 \%)$ \\
\hline$\geq 1$ vs 0 & $97.2 \%(85.5 \%$ to $99.9 \%)$ & $3.2 \%(1.2 \%$ to $6.8 \%)$ & $85.7 \%(42.1 \%$ to $99.6 \%)$ & $16.1 \%(11.4 \%$ to $21.6 \%)$ \\
\hline \multicolumn{5}{|l|}{ Colposcope } \\
\hline 10 vs $<10$ & $0.0 \%(0.0 \%$ to $9.7 \%)$ & $99.5 \%(97.1 \%$ to $100.0 \%)$ & $83.9 \%(78.5 \%$ to $88.5 \%)$ & $0.0 \%$ (0.0\% to $97.5 \%)$ \\
\hline$\geq 9$ vs $<9$ & $11.1 \%(3.1 \%$ to $26.1 \%)$ & $96.8 \%(93.2 \%$ to $98.8 \%)$ & $85.1 \%(79.6 \%$ to $89.6 \%)$ & $40.0 \%(12.2 \%$ to $73.8 \%)$ \\
\hline$\geq 8$ vs $<8$ & $30.6 \%(16.3 \%$ to $48.1 \%)$ & $90.5 \%(85.4 \%$ to $94.3 \%)$ & $87.2 \%(81.7 \%$ to $91.6 \%)$ & $37.9 \%(20.7 \%$ to $57.7 \%)$ \\
\hline$\geq 7$ vs $<7$ & $44.4 \%(27.9 \%$ to $61.9 \%)$ & $81.0 \%(74.6 \%$ to $86.3 \%)$ & $88.4 \%(82.7 \%$ to $92.8 \%)$ & $30.8 \%(18.7 \%$ to $45.1 \%)$ \\
\hline$\geq 6$ vs $<6$ & $52.8 \%(35.5 \%$ to $69.6 \%)$ & $66.1 \%(58.9 \%$ to $72.8 \%)$ & $88.0 \%(81.5 \%$ to $92.9 \%)$ & $22.9 \%(14.4 \%$ to $33.4 \%)$ \\
\hline$\geq 5$ vs $<5$ & $66.7 \%(49.0 \%$ to $81.4 \%)$ & $45.0 \%(37.7 \%$ to $52.4 \%)$ & $87.6 \%(79.4 \%$ to $93.4 \%)$ & $18.8 \%(12.4 \%$ to $26.6 \%)$ \\
\hline$\geq 4$ vs $<4$ & $83.3 \%(67.2 \%$ to $93.6 \%)$ & $22.2 \%(16.5 \%$ to $28.8 \%)$ & $87.5 \%(74.8 \%$ to $95.3 \%)$ & $16.9 \%(11.7 \%$ to $23.3 \%)$ \\
\hline$\geq 3$ vs $<3$ & $91.7 \%(77.5 \%$ to $98.2 \%)$ & $14.3 \%(9.6 \%$ to $20.1 \%)$ & $90.0 \%(73.5 \%$ to $97.9 \%)$ & $16.9 \%(11.9 \%$ to $22.9 \%)$ \\
\hline$\geq 2$ vs $<2$ & $94.4 \%(81.3 \%$ to $99.3 \%)$ & $6.9 \%(3.7 \%$ to $11.5 \%)$ & $86.7 \%(59.5 \%$ to $98.3 \%)$ & $16.2 \%(11.5 \%$ to $21.9 \%)$ \\
\hline$\geq 1$ vs 0 & $97.2 \%(85.5 \%$ to $99.9 \%)$ & $3.2 \%(1.2 \%$ to $6.8 \%)$ & $85.7 \%(42.1 \%$ to $99.6 \%)$ & $16.1 \%(11.4 \%$ to $21.6 \%)$ \\
\hline
\end{tabular}

within colposcopy and with the Gynocular is also a feasible and safe opportunity to lessen the human resource gap within colposcopy in low-resource settings. This pragmatic but also highly accurate approach may have widespread implications to lower the epidemic high incidence of cervical cancer.

Bowring $e a^{13}$ showed that trainee unaccredited colposcopists were as accurate as accredited colposcopists in detecting cervical lesions using the Swede score, findings analogous to our findings of VIA nurse colposcopists compared to accredited doctor colposcopists. Our study also showed that a VIA nurse colposcopist Swede score of 8 or above had parallel high specificities of CIN2+ as the Swede score of the doctors. These results are comparable to CIN2+ specificities in Swede score trials by doctors from both high-resource ${ }^{1112}$ and low-resource settings. ${ }^{13} 14$

The Swede score colposcopy system works well with various healthcare professionals and economical settings.

It has been suggested ${ }^{13-15}$ that the Swede score may be used as a primary cervical screening as well as a see and treat method of cervical lesions in low-resource

Table 3 Sensitivity and specificity for different cut-off levels for CIN 2+ (CIN 2, CIN 3 and invasive cervical cancer (doctors, $n=228)$ )

\begin{tabular}{ccccc}
\hline & Sensitivity (95\% Cl) & Specificity (95\% Cl) & PPV (95\% Cl) & NPV (95\% Cl) \\
\hline Gynocular & & & & \\
10 vs $<10$ & $5.6 \%(0.7 \%$ to $18.7 \%)$ & $97.4 \%(94.0 \%$ to $99.1 \%)$ & $84.5 \%(79.1 \%$ to $89.1 \%)$ & $28.6 \%(3.7 \%$ to $71.0 \%)$ \\
$\geq 9$ vs $<9$ & $22.2 \%(10.1 \%$ to $39.2 \%)$ & $93.7 \%(89.3 \%$ to $96.7 \%)$ & $86.5 \%(81.0 \%$ to $90.8 \%)$ & $40.0 \%(19.1 \%$ to $63.9 \%)$ \\
$\geq 8$ vs $<8$ & $36.1 \%(20.8 \%$ to $53.8 \%)$ & $88.0 \%(82.5 \%$ to $92.2 \%)$ & $88.0 \%(82.5 \%$ to $92.2 \%)$ & $36.1 \%(20.8 \%$ to $53.8 \%)$ \\
$\geq 7$ vs $<7$ & $52.8 \%(35.5 \%$ to $69.6 \%)$ & $74.9 \%(68.1 \%$ to $80.9 \%)$ & $89.4 \%(83.5 \%$ to $93.7 \%)$ & $28.4 \%(18.0 \%$ to $40.7 \%)$ \\
$\geq 6$ vs $<6$ & $61.1 \%(43.5 \%$ to $76.9 \%)$ & $52.9 \%(45.5 \%$ to $60.1 \%)$ & $87.8 \%(80.4 \%$ to $93.2 \%)$ & $19.6 \%(12.7 \%$ to $28.2 \%)$ \\
$\geq 5$ vs 4 & $83.3 \%(67.2 \%$ to $93.6 \%)$ & $22.0 \%(16.3 \%$ to $28.5 \%)$ & $87.5 \%(74.8 \%$ to $95.3 \%)$ & $16.8 \%(11.6 \%$ to $23.1 \%)$ \\
Colposcope & & & & \\
10 vs $<10$ & $5.6 \%(0.7 \%$ to $18.7 \%)$ & $97.4 \%(94.0 \%$ to $99.1 \%)$ & $84.5 \%(79.1 \%$ to $89.1 \%)$ & $28.6 \%(3.7 \%$ to $71.0 \%)$ \\
$\geq 9$ vs $<9$ & $19.4 \%(8.2 \%$ to $36.0 \%)$ & $93.2 \%(88.6 \%$ to $96.3 \%)$ & $86.0 \%(80.5 \%$ to $90.4 \%)$ & $35.0 \%(15.4 \%$ to $59.2 \%)$ \\
$\geq 8$ vs $<8$ & $36.1 \%(20.8 \%$ to $53.8 \%)$ & $88.0 \%(82.5 \%$ to $92.2 \%)$ & $88.0 \%(82.5 \%$ to $92.2 \%)$ & $36.1 \%(20.8 \%$ to $53.8 \%)$ \\
$\geq 7$ vs $<7$ & $52.8 \%(35.5 \%$ to $69.6 \%)$ & $75.4 \%(68.7 \%$ to $81.3 \%)$ & $89.4 \%(83.6 \%$ to $93.7 \%)$ & $28.8 \%(18.3 \%$ to $41.3 \%)$ \\
$\geq 6$ vs $<6$ & $61.1 \%(43.5 \%$ to $76.9 \%)$ & $53.4 \%(46.1 \%$ to $60.6 \%)$ & $87.9 \%(80.6 \%$ to $93.2 \%)$ & $19.8 \%(12.9 \%$ to $28.5 \%)$ \\
$\geq 5$ vs 4 & $83.3 \%(67.2 \%$ to $93.6 \%)$ & $22.5 \%(16.8 \%$ to $29.1 \%)$ & $87.8 \%(75.2 \%$ to $95.4 \%)$ & $16.9 \%(11.7 \%$ to $23.2 \%)$ \\
\hline PPV, positive predictive value; NPV, negative predictive value. & &
\end{tabular}




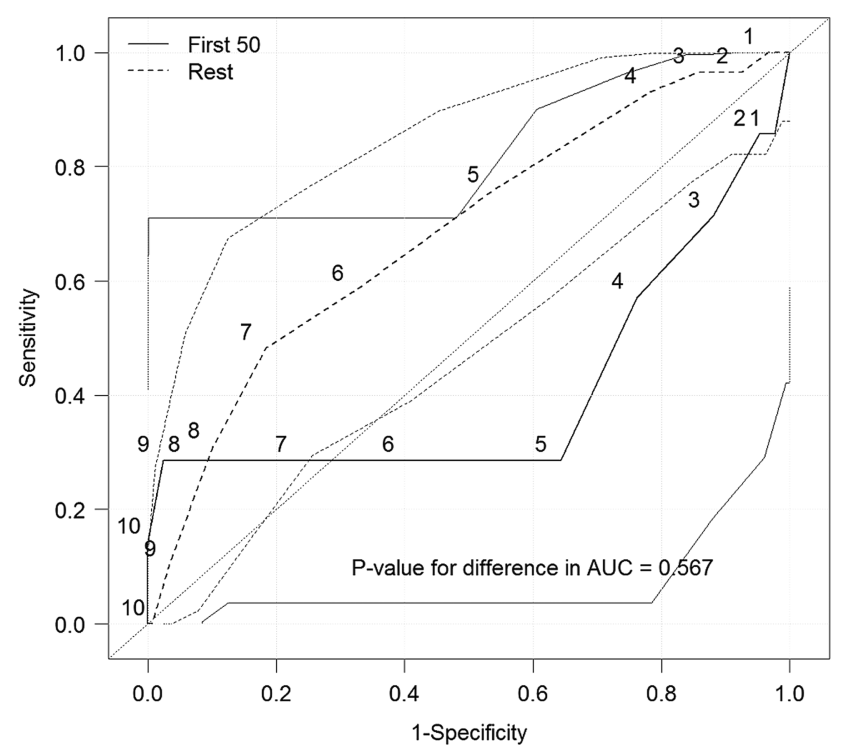

Figure 6 Receiver operating characteristic curves for predicting a positive biopsy result CIN2+(CIN 2, CIN 3 and invasive cervical cancer) using a Gynocular (Nurses first 50 vs the rest).

settings. Thus, it is interesting to note that Swede score directed punch biopsies in women with a score of 4 and above were more accurate than cytology in detecting CIN2+ lesions than cytology in VIA positive women in Uganda and Bangladesh. ${ }^{14} 15$ However, our study show that it is needed to further validate the Swede score's sensitivity and specificity to detect CIN2+ in low-resource populations, possible with biopsies from Swede score 0 in screening naïve, VIA positive women as well as human papillomavirus status. This is important as the Swede score was previously validated in women with an abnormal referral cytology in high-resource settings. ${ }^{12} 13$

Moreover, in a multicentre randomised controlled trial, ${ }^{28}$ direct colposcopy identified more cervical lesions than repeat cytology and studies from Bangladesh and Nigeria ${ }^{20} 29$ concluded that the immediate 'see and treat' protocol after colposcopic examination of highgrade CIN was cheaper, less time consuming and more effective with less complication and good compliance. It reduced the number of visits to the clinic and failure to receive treatment associated with good compliance. ${ }^{20} 29$

A recent trial from Bangladesh summarised that VIA should be used as the primary screening tool, although itss low sensitivity and specificity due to the limitations of a low-resource country, and the high false-positive results and overtreatment can be minimised by colposcopy evaluation of the VIA positive women. ${ }^{30}$ Also, a notable response was observed from women at the Upazila (subdistrict) level on the days of VIA Camps, indicating the positive attitude of women of having an examination for prevention of a disease. ${ }^{31}$ VIA camps had a synergistic effect on the community, as women attending the VIA camp on the first and second days informed other women about the VIA test and availability of the service at the Upazila Health Complex (UHC). ${ }^{32}$ Thus, a combination VIA camp and 'treatment camp' may be useful in remote rural areas of low-resource settings; by administering a 'See and treat' protocol with Gynocular, it could offer an attractive option for a successful screening outcome. A mobile van equipped with an examination chair, Gynocular and loop electrosurgical excision procedure could offer a future approach in rural areas of remote districts. This will also reduce travel costs and loss of working days for the VIA positive women not requiring treatment. However, expertise on colposcopy needs to be emphasised to reduce overtreatment, and further research on nurse-led Swede score colposcopy in lowresource settings and a learning curve are needed. Improvement of patients' knowledge and proper counselling should be important components of this protocol. Women would then not have to accept treatment only on the basis of the VIA result and risk the associated drawbacks of overtreatment. A similar benefit is applicable to other developing countries like Bangladesh.

There is now an opportunity for policymakers to reduce the human resource gap by organising training programmes for educating nurse colposcopists and outreach see-and-treat teams in low-resource settings, and to promote and evaluate such programmes in -adequately powered research studies. Sankaranarayanan et $a l^{31}$ showed previously that adequately trained nurses can be used to deliver colposcopy and cryotherapy services and are important, reliable and efficient alternate human resources. Training of nurses on colposcopy and the use of the Gynocular could have a widespread effect in reducing the number of women dying from cervical cancer, which in turn could have a major impact on community structure and wealth.

Future research should further evaluate VIA screening and direct colposcopy in low-resource settings.

\section{CONCLUSION}

In summary, a Swede score colposcopy examination by a nurse or doctor colposcopist, using the Gynocular or stationary colposcope, is similarly good in detecting cervical lesions.

The Gynocular offers an opening for reducing the human resource gap in low-resource settings by offering nurse-colposcopist teams a tool for early detection of cervical high-grade lesions. Nurse-colposcopy in low resource settings with the Gynocular and the Swede score should be further explored and evaluated.

\section{Author affiliations}

${ }^{1}$ Department of Obstetrics and Gynecology, Bangabandhu Sheikh Mujib Medical University (BSMMU), Dhaka, Bangladesh

${ }^{2}$ Department of Obstetrics and Gynaecology, Green life Medical College Hospital, Dhaka, Bangladesh

${ }^{3}$ Departments of Obstetrics and Gynecology and Clinical Sciences, Karolinska Institutet, Danderyd Hospital, Stockholm, Sweden

${ }^{4}$ Statisticon, Uppsala, Sweden

${ }^{5}$ Karolinska Institutet, Karolinska University Hospital, Stockholm, Sweden 
Acknowledgements The authors would like to thank all the women who participated in the study. The authors would also like to thank the doctors and nurses who examined the women at the colposcopy clinic of Bangabandhu Sheikh Mujib Medical University in Dhaka, Bangladesh. The authors would also like to thank the BSMMU in Bangladesh and Karolinska Institutet in Sweden for allowing us to perform the study at their premises. The authors wish to thank H\&M Concious Foundation's funding for the study.

Contributors EAWS and IS initiated the project. EAWS and AN designed the study. AN, JSR, MAC, QK, RA, MT and EAWS provided gynaecological expertise. AN, JSR, MAC, QK, RA and CW collected the data. MT, IS and MT provided statistical expertise. All authors performed literature searches and wrote and edited the article. All authors contributed to analysis of the data.

Funding MT received financial support for the submitted work from Gynius $A B$ for statistical expertise. CW received financial support for study support in Bangladesh. H\&M Conscious Foundation and Gynius AB funded the research project.

Competing interests EAWS and IS are shareholders in Gynius. H\&M Conscious Foundation and Gynius AB funded the research project. EAWS is the inventor of the Gynococular.

Patient consent Obtained.

Ethics approval This study was approved by the research ethics committee of Bangabandhu Sheikh Mujib Medical University (BSMMU) in Dhaka, Bangladesh and the Karolinska Institutet, Stockholm, Sweden. All participants provided informed consent.

Provenance and peer review Not commissioned; externally peer reviewed.

Data sharing statement The data set is available on request from the corresponding author.

Open Access This is an Open Access article distributed in accordance with the Creative Commons Attribution Non Commercial (CC BY-NC 4.0) license, which permits others to distribute, remix, adapt, build upon this work noncommercially, and license their derivative works on different terms, provided the original work is properly cited and the use is non-commercial. See: http:// creativecommons.org/licenses/by-nc/4.0/

\section{REFERENCES}

1. Ferlay J, Soerjomataram I, Ervik M, et al. GLOBOCAN 2012 v1.0, cancer incidence and mortality worldwide: IARC cancer base no. 11 [Internet]. Lyon, France: International Agency for Research on Cancer, 2013

2. Castellsagué X, Sanjosé S, Aguado T, et al. GLOBOCAN: Cervical Cancer Incidence and Mortality Worldwide in 2008. CANCER FACT SHEET, 2008.

3. Anorlu RI. What is the significance of the HPV epidemic? Can J Urol 2008;15:3860-5.

4. Vaccarella S, Lortet-Tieulent J, Plummer M, et al. Worldwide trends in cervical cancer incidence: impact of screening against changes in disease risk factors. Eur J Cancer 2013;49:3262-73.

5. Garcia M, Jemal A, Ward EM, et al. Global cancer facts and figures 2007. American Cancer Society, 2007

6. Nessa A, Hussain MA, Rahman JN, et al. Screening for cervical neoplasia in Bangladesh using visual inspection with acetic acid. Int J Gynaecol Obstet 2010;111:115-18.

7. Sahasrabuddhe VV, Parham GP, Mwanahamuntu MH, et al. Cervical cancer prevention in low- and middle-income countries: feasible, affordable, essential. Cancer Prev Res (Phila) 2012;5:11-17.

8. Sauvaget C, Fayette JM, Muwonge R, et al. Accuracy of visua inspection with acetic acid for cervical cancer screening. Int $J$ Gynaecol Obstet 2011;113:14-24

9. Arbyn M, Kyrgiou M, Simoens C, et al. Perinatal mortality and other severe adverse pregnancy outcomes associated with treatment of cervical intraepithelial neoplasia: meta-analysis. BMJ 2008;337:a1284.

10. Underwood M, Arbyn M, Parry-Smith W, Accuracy of colposcopy-directed punch biopsies: a systematic review and meta-analysis. BJOG 2012;119:1293-301.
11. Pimple SA, Amin G, Goswami S, et al. Evaluation of colposcopy vs cytology as secondary test to triage women found positive on visual inspection test. Indian J Cancer 2010;47:308-13.

12. Strander B, Ellström-Andersson A, Franzén S, et al. The performance of a new scoring system for colposcopy in detecting high-grade dysplasia in the uterine cervix. Acta Obstet Gynecol Scand 2005;84:1013-17.

13. Bowring J, Strander B, Young M, et al. The Swede score: evaluation of a scoring system designed to improve the predictive value of colposcopy. J Low Genit Tract Dis 2010;14:301-5.

14. Ngonzi J, Bajunirwe F, Wistrand C, et al. Agreement of colposcope and Gynocular in assessment of cervical lesions by Swede score: a randomized, crossover pilot trial. J Low Genit Tract Dis 2013;17:372-7.

15. Nessa A, Wistrand C, Begum SA, et al. Evaluation of stationary colposcope and the gynocular, by the Swede score systematic colposcopic system in VIA positive women: a crossover randomized trial. Int J Gynecol Cancer 2014;24:339-45.

16. Kilic G, England J, Borahay M, et al. Accuracy of physician and nurse practitioner colposcopy to effect improved surveillance of cervical cancer. Eur J Gynaecol Oncol 2012;33:183-6.

17. McPherson G, Horsburgh M, Tracy C. A clinical audit of a nurse colposcopist. Colposcopy: cytology: histology correlation. Nurs Prax N Z2005;21:13-23.

18. Hartz LE. Quality of care by nurse practitioners delivering colposcopy services. J Am Acad Nurse Pract 1995;7:23-7.

19. Ahmed T, Ashrafunnessa, Rahman J. Development of a visual inspection programme for cervical cancer prevention in Bangladesh. Reprod Health Matters 2008;16:78-85.

20. Nessa A, Rashid MH, E-Ferdous N, et al. Screening for and management of high-grade cervical intraepithelial neoplasia in Bangladesh: a cross-sectional study comparing two protocols. J Obstet Gynaecol Res 2013;39:564-7.

21. Cox JT. More questions about the accuracy of colposcopy: what does that mean for cervical cancer prevention? Obstet Gynecol 2008;111:1266.

22. Richart RM. A modified terminology for cervical intraepithelial neoplasia. Obstet Gynecol 1990;75:131-3.

23. R Development Core Team. R: A language and environment for statistical computing. Vienna, Austria: R Foundation for Statistical Computing, 2011. ISBN: 3-900051-07-0. http://www.R-project.org/

24. Landis JR, Koch GG. The measurement of observer agreement for categorical data. Biometrics 1977;33:159-74.

25. Hill K, Thomas K, AbouZahr C, et al. Estimates of maternal mortality worldwide between 1990 and 2005: an assessment of available data. Lancet 2007;370:1311-19.

26. Basu PS, Sankaranarayanan R, Mandal R, et al.; Calcutta Cervica Cancer Early Detection Group. Visual inspection with acetic acid and cytology in the early detection of cervical neoplasia in Kolkata, India. Int J Gynecol Cancer 2003;13:626-32.

27. FIGO Safe Motherhood and Newborn Health Committee. Human resources for health in the low-resource world: Collaborative practice and task-shifting in maternal and neonatal care. Int J Gynecol Obstet 2009;105:74-6.

28. Hofmeyr GJ, Haws RA, Bergström S, et al. Obstetric care in low-resource settings: what, who, and how to overcome challenges to scale up?. Int J Gynaecol Obstet 2009;107(Suppl 1): S21-44.

29. Cruickshank M, Murray G, Parkin D, et al. Cytological surveillance compared with immediate referral for colposcopy in management of women with low grade cervical abnormalities: multicentre randomised controlled trial. TOMBOLA Group. BMJ 2009;339: b2546.1.

30. Chigbu CO, Onyebuchi AK. See-and-treat management of high-grade squamous intraepithelial lesions in a resource-constrained African setting. Int J Gynaecol Obstet 2013;124:204-6.

31. Nessa A, Nahar KN, Begum SA, et al. Comparison between Visual Inspection of Cervix and Cytology Based Screening Procedures in Bangladesh. Asian Pac J Cancer Prev 2013;14:7607-11.

32. Nessa A, Hussain MA, Rashid MH, et al. Role of print and audiovisual media in cervical cancer prevention in Bangladesh. Asian Pac J Cancer Prev 2013;14:3131-7.

33. Sankaranarayanan R, Rajkumar R, Esmy PO, et al. Effectiveness, safety and acceptability of 'see and treat' with cryotherapy by nurses in a cervical screening study in India. $\mathrm{Br} \mathrm{J}$ Cancer 2007;96:738-43. 